\title{
Mixed Mullerian tumor: a case report
}

\author{
Mahrukh Nazneen*, Uma N. Wankhede \\ Department of Obstetrics and Gynecology, B.J.G.M.C. and S.G.H. Pune, Maharashtra, India
}

Received: 24 August 2017

Accepted: 25 September 2017

\section{*Correspondence:}

Dr. Mahrukh Nazneen,

E-mail: mahrukh2010@gmail.com

Copyright: $\odot$ the author(s), publisher and licensee Medip Academy. This is an open-access article distributed under the terms of the Creative Commons Attribution Non-Commercial License, which permits unrestricted non-commercial use, distribution, and reproduction in any medium, provided the original work is properly cited.

\begin{abstract}
Malignant mixed Müllerian tumor, also known as malignant mixed mesodermal tumor, MMMT and carcinosarcoma, is a malignant neoplasm found in the uterus, the ovaries, the fallopian tubes and other parts of the body that contains both carcinomatous (epithelial tissue) and sarcomatous (connective tissue) components. A 50-year female P1L1A1 with complaints of pervaginum bleeding and pain in abdomen was diagnosed to have carcinosarcoma endometrium for which hysterectomy with bilateral salphingo-opherectomy was done.
\end{abstract}

Keywords: Carcinosarcoma, Hysterectomy, Malignant mixed Mullerian tumor

\section{INTRODUCTION}

Malignant mixed Müllerian tumor, also known as malignant mixed mesodermal tumor, MMMT and carcinosarcoma, is a malignant neoplasm found in the uterus, the ovaries, the fallopian tubes and other parts of the body that contains both carcinomatous (epithelial tissue) and sarcomatous (connective tissue) components. It is divided into two types, homologous (in which the sarcomatous component is made of tissues found in the uterus such as endometrial, fibrous and/or smooth muscle tissues) and a heterologous type (made up of tissues not found in the uterus, such as cartilage, skeletal muscle and/or bone). MMMT account for between two and five percent of all tumours derived from the body of the uterus, and are found predominantly in postmenopausal women with an average age of 66 years. Risk factors are similar to those of adenocarcinoma uterus and include obesity, exogenous oestrogen therapies, and nulliparity. Less well-understood but potential risk factors include tamoxifen therapy and pelvic irradiation. ${ }^{1}$ A 50-year female P1L1A1 with complaints of pervaginum bleeding and pain in abdomen was diagnosed to have carcinosarcoma endometrium for which hysterectomy with bilateral salphingo-opherectomy was done.

\section{CASE REPORT}

A 50-year female P1L1A1 with previous LSCS, tubectomized 11 years back, resident of Namdev Nagar, Wadgaosheri came to emergency ward with chief complaints of pain in abdomen and per vaginum bleeding on and off for 1 month, no history of per vagium discharge. On detailed evaluation patient gives the history of 6 months amenorrhea followed by bleeding per vaginum for 1 month soaking 4 pads per day associated with passage of clots. Investigations were done with Transabdominal sonography showing bulky uterus thickened and irregular endometrium with multiple cystic changes causing thinning of myometrium with significant peripheral vascularity suggestive of malignant neoplastic etiology - carcinoma endometrium. Dilatation and curettage was done for tissue diagnosis with histopathology report stating no typing possible, after which tissue block was sent for review examination from senior pathologist which was suggestive of carcinosarcoma (malignant mixed Mullerian tumor). Patient was followed with CT (abdomen + pelvis) which showed bulky uterus $16.6 \times 9.4 \times 8.8 \mathrm{~cm}$ with heterogeneously enhancing polypoidal mass lesion involving uterine cavity approx. $8.7 \times 5.4 \times 5.2 \mathrm{~cm}$. 
Intramural fibroid $3.4 \times 4.1 \mathrm{~cm}$ involving left lower wall. Subserosal fibroid $1.4 \times 3 \mathrm{~cm}$ involving right lower lateral wall. Both ovaries are visualized and normal, bowel and bladder appears normal. Oncoreference was done and hysterectomy was advised with follow-up USG. Preanesthetic checkup for the patient was done with all routine blood investigations and patient was posted for abdominal hysterectomy with bilateral salphingo oophorectomy, lymph node dissection was done and specimen sent for histopathological examination. Post hysterectomy histopathological report was suggestive of malignant mixed Mullerian tumor without local spread, Tumor not infiltrating myometrium muscle fibers, section studied from parametrium shows adipose tissue free of tumor, no evidence of pelvic and paraaortic lymph node involvement. Ultrasonography was done with no significant abnormality. Review oncoreference was done and pelvic radiation was advised with close follow up, patient was discharged on request after stitch removal and advised follow up after 7 days, patient however lost follow up.

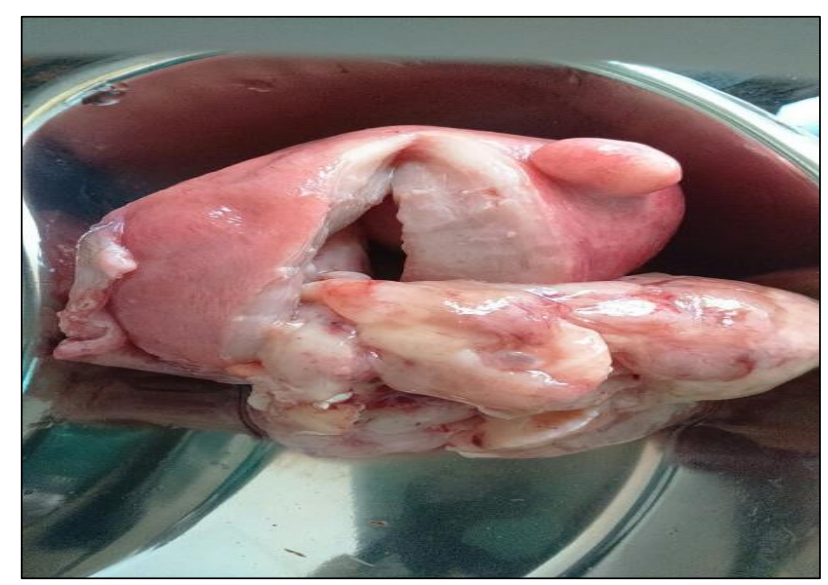

Figure 1: Specimen showing uterus with polypoid tumor.

\section{DISCUSSION}

Malignant uterine neoplasm's containing both carcinomatous and sarcomatous elements are designated in the World Health Organisation (WHO) classification of uterine neoplasm's as carcinosarcomas. Gebhardt in 1899 appears to have reported the first case, Meyer, after a personal examination of the slides, accepted it as authentic. ${ }^{3}$ Carcinosarcomas representing less than $5 \%$ of all uterine tumors, account for $16.4 \%$ of all deaths caused by a uterine malignancy. ${ }^{4}$ MMMT has been identified in decreasing order of frequency in the vagina, cervix, and ovary and most rarely in the fallopian tubes. ${ }^{5-8}$ There are three main theories regarding the histogenesis of MMMT namely:

- The collision theory suggests that the carcinoma and sarcoma are two independent neoplasms. ${ }^{9,10}$

- The combination theory suggest that both components and derived from a single stem cell that undergoes divergent differentiation early in the evolution of the tumor.

- The conversion theory suggests that the sarcomatous elements derive from the carcinoma during the evolution of the tumor.

On exploring the literature, we found that $\mathrm{W} G$ McCluggage named one more theory: The composition theory suggest that the spindle cell component is a pseudosarcomatous stromal reaction to the presence of the carcinoma.

Outcome of MMMTs is determined primarily by depth of invasion and stage. As with endometrial carcinomas, the prognosis is influenced by the grade and type of the adenocarcinoma, being poorest with serous differentiation. MMMTs are highly malignant; a stage I tumor has an expected five-year survival rate of 50\%, while the overall five-year survival rate is less than $20 \%$. $^{1}$

According to FIGO staging, uterine carcinosarcoma is staged as carcinoma of uterus.

- IA Tumor confined to the uterus, no or $<1 / 2$ myometrial invasion

- IB Tumor confined to the uterus, $>1 / 2$ myometrial invasion

- II Cervical stromal invasion, but not beyond uterus

- $\quad$ IIIA Tumor invades serosa or adnexa

- IIIB Vaginal and/or parametrial involvement

- $\quad$ IIIC1 Pelvic node involvement

- IIIC2 Para-aortic involvement

- IVA Tumor invasion bladder and/or bowel mucosa

- IVB Distant metastases including abdominal metastases and/or inguinal lymph nodes.

Diagnosis of MMMT is most often made postoperatively by histopathological examination and Immunohistochemical (IHC) studies. Microscopically MMMT is composed of both epithelial and mesenchymal elements may be intermittently mixed or be seen as two distinct components. ${ }^{12}$ The mesenchymal elements may be (a) homologous, containing cells native to the uterus including stromal sarcoma, fibrosarcoma or leiomyosarcoma (2\%) or (b) heterologous with mixed components including rhabdomyosarcoma (18\%), chondrosarcoma (10\%), osteosarcoma $(5 \%)$ or liposarcoma $(1 \%){ }^{2}$ Several studies have found concordance of p53 staining between the carcinomatous and sarcomatous components in MMMT. ${ }^{13,14}$

IHC studies have shown that both the sarcomatous and carcinomatous components often coexpress cytokeratin and vimentin. ${ }^{15}$ In the present case, microscopic sections studied showed haphazardly admixed of both epithelial and mesenchymal components. The mesenchymal component showed homologous spindle cell sarcomatous component displaying marked pleomorphism and atypical mitotic activity, suggestive of malignant mixed 
Mullerian tumor without local spread, tumor not infiltrating myometrium muscle fibres, section studied from parametrium shows adipose tissue free of tumor, no evidence of pelvic and paraaortic lymph node involvement so stage IA.

\section{CONCLUSION}

To conclude MMMT which arise from female genital tract can have both epithelial and mesenchymal component. If epithelial component is benign and mesenchymal component is malignant then it is called as Adenosarcoma. If both epithelial component and mesenchymal component is malignant then it is called as Carcinosarcoma. Malignant mixed Mullerian tumour is a rare, highly aggressive, rapidly progressive neoplasm associated with a poor prognosis.

\section{Funding: No funding sources}

Conflict of interest: None declared

Ethical approval: Not required

\section{REFERENCES}

1. Brooks SE, Zhan $M$, Cote $T$, Baquet $C R$. Surveillance, epidemiology, and end results analysis of 2677 cases of uterine sarcoma 1989-1999. Gynecol Oncol. 2004;93:204-8.

2. El-Nashar SA, Mariani A. Uterine carcinosarcoma. Clin Obstet Gynecol. 2011;54(2):292-304

3. Meyer R. In Veit-Stoeckel. 1930;6:770-4.

4. Bosquet JS, Terstrie SA, Cliby WA, Brown-Jones M, Kaur JS, Podratz KC, et al. The impact of multimodal therapy on survival for uterine carcinosarcomas. Gynecol Oncol. 2010;116(3):41923.

5. Ahuja A, Safaya R, Prakash G, Kumar L, Shukla, NK. Primary mixed Mullerian tumor of the vagina: a case report with review of the literature. Pathology Research and Practice. 2011;207(4):253-5.

6. Sharma NK, Sorosky JI, Bender D, Fletcher MS, Sood AK. Malignant mixed Mullerian tumor
(MMMT) of the cervix. Gynecol Oncol. 2005;97(2):442-5.

7. Duman BB, Kara IO, Gunaldi M, Ercolak V. Malignant mixed Mullerian tumor of the ovary with two cases and review of the literature. Arch Gynecol Obstet. 2011;283(6):1363-8.

8. Shen YM, Xie YP, Xu L. Malignant mixed Mullerian tumor of the fallopian tube: report of two cases and review of literature. Arch Gynecol Obstet. 2010;281(6):1023-8.

9. de Jong RA, Nijman HW, Wijbrandi TF, Reyners AK, Boezen HM, Hollema H. Molecular markers and clinical behavior of uterine carcinosarcomas: focus on the epithelial tumor component. Modern Pathology. 2011;24(10):1368.

10. Jin Z, Ogata S, Tamura G. Carcinosarcomas (malignant Mullerian mixed tumors) of the uterus and ovary: a genetic study with special reference to histogenesis. Int J Gynecol Pathol. 2003;22(4):36873.

11. McCluggage WG. Malignant biphasic uterine tumors: carcinosarcomas or metaplastic carcinomas? J Clin Pathol. 2002;55:321-5.

12. Brown L. Pathology of uterine malignancies. Clin Oncol. 2008;20(6):433-47.

13. Mayall F, Rutty K, Campbell F. p53 immunostaining suggests that uterine carcinosarcomas are monoclonal. Histopathol. 1994:24:211-4.

14. Szukala SA, Marks JR, Burchette JL. Coexpression of p53 by epithelial and stromal elements in carcinosarcoma of the female genital tract: an immunohistochemical study of 19 cases. Int $\mathbf{J}$ Gynecol Cancer. 1999;9:131-6.

15. George E, Manivel JC, Dehner LP. Malignant mixed Mullerian tumors: an immunohistochemical study of 47 cases, with histogenetic considerations and clinical correlation. Hum Pathol. 1991;22:215-23.

Cite this article as: Nazneen M, Wankhede UN. Mixed Mullerian tumor: a case report. Int J Reprod Contracept Obstet Gynecol 2017;6:5161-3. 